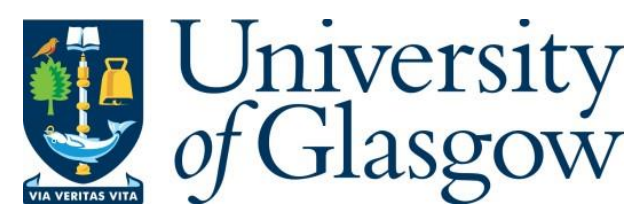

Lee, J., Lamarche, M. and Georgiev, V. P. (2019) The First-Priniple Simulation Study on the Specific Grain Boundary Resistivity in Copper Interconnects. In: 2018 IEEE 13th Nanotechnology Materials \& Devices Conference (NMDC 2018), Portland, OR, USA, 14-17 Oct 2018, ISBN 9781538610169.

There may be differences between this version and the published version. You are advised to consult the publisher's version if you wish to cite from it.

http://eprints.gla.ac.uk/167521/

Deposited on: 23 August 2018

Enlighten - Research publications by members of the University of Glasgow http://eprints.gla.ac.uk 


\section{The First-Priniple Simulation Study on the Specific Grain Boundary Resistivity in Copper Interconnects}

\author{
Jaehyun Lee \\ School of Engineering \\ University of Glasgow \\ Glasgow, United Kingdom \\ Jaehyun.Lee@glasgow.ac.uk
}

\author{
Michel Lamarche \\ School of Engineering \\ University of Glasgow \\ Glasgow, United Kingdom \\ 2122265L@student.gla.ac.uk
}

\author{
Vihar P. Georgiev \\ School of Engineering \\ University of Glasgow \\ Glasgow, United Kingdom \\ Vihar.Georgiev@glasgow.ac.uk
}

\begin{abstract}
In this work, we present a systematic simulation study of numerous copper $(\mathrm{Cu})$ grain boundaries with the nonequilibrium Green's function (NEGF) framework based on the Density Functional Theory (DFT). In order to evaluate the effect of specific resistivity of various grain boundary profiles we developed the required methodology and we proposed an analytical equation for predicting the specific resistivity at each GB configuration. Moreover, in this work we also considered different crystal transport orientations and coincidence site lattices. Based on our simulations, we found that the specific grain boundary resistivity strongly depends on the transport orientations of the grains but not on the coincidence site lattice (CSL) density.
\end{abstract}

Keywords- $\mathrm{Cu}$ interconnect, Grain boundary, Density Functional Theory, simulations and modelling

\section{INTRODUCTION}

Due to a recent aggressive downscaling of transistors in integrated circuit (IC) chips, the interconnects reached a nanoscale size which makes the quantum mechanical effect significantly much more pronounced in comparison to the previous technology nodes. Hence, scaling down the copper $\mathrm{Cu}$ interconnects to tens of nanometres is inevitable. This creates significant problems for the semiconductor industry that needs to accommodate, e.g. the increasing of the circuit delay [1]. Moreover, the lower level of interconnects is the most vulnerable because they have the smallest dimension in comparison to all other levels of interconnects. This leads to an increase in the capacitance between metal lines and, hence, the resistivity. This occurs mainly due to the presence of the grain boundary (GB) scattering and the surface roughness (SR) scattering [2-4]. The increase in the resistivity also causes reliability problems, such as electromigration $[5,6]$, which have a detrimental effect on the interconnects. Hence, understanding in detail effects such as electromigration, GB and SR scatterings could solve some of the physical and technical challenges, which the industry faces today. The purpose of this work is to address some of those problems and more specifically the effect of the GB scattering on the resistivity in the nano-scale interconnects.

It is well-known that the specific resistivity at GB $\left(\gamma_{R}\right)$ strongly depends on the atomistic structures, such as specific

This work is supported by the U.K. EPSRC under Project EP/P009972/1 and Project EP/S001131/1. atomic configuration and crystal orientation [7]. Experimental measurement of the atomic configuration at nanoscale dimension is very challenging and the best way to evaluate and predict the GB resistivity is to perform numerical simulations. For example, in 2014, César et al. performed the Density Functional Theory (DFT) simulations to calculate $\gamma_{\mathrm{R}}$ with the $\Sigma 3, \Sigma 5, \Sigma 9, \Sigma 11, \Sigma 13$ a and $\Sigma 17$ a grain boundaries [7]. Herein $\Sigma$ indicates the reciprocal coincidence site lattice (CSL) density. Although there are many possible atomistic structures having different transport orientations for each $\Sigma$, they selected and studied one case, specifically the one with the fewest atoms. Thanks to the four-probe scanning tunnelling microscopy, the specific resistivity of twin GBs was also measured and compared to the simulation results [8].

In this work, we calculate the specific resistivity of many GB configurations by using a DFT - Non-Equilibrium Green's Function (NEGF) framework [9]. The studied structures are limited to relatively small sizes containing single grain boundaries and less than a few hundred atoms because of the computational burden required to perform the DFT-NEGF calculations. However, we believe that our simulation results are sufficient to propose and develop a methodology and analytical equation to predict the specific resistivity of different

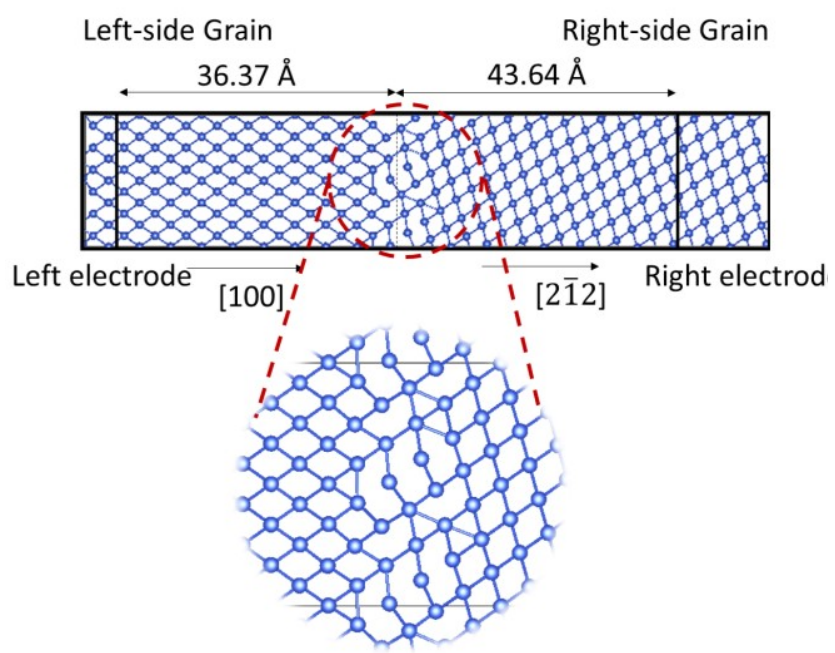

Fig. 1 An example of the atomistic structure of a twin GB (case \#1 in Table 1). A box indicates the DFT simulation box. A blue sphere is a $\mathrm{Cu}$ atom. 
types of GBs. The paper is organised in the following way. Section II presents the main characteristics of our simulation approach, which includes the generation of the atomic structure and evaluation of the ballistic conductance and specific resistivity at the GB. In Section III, we discuss our simulation results, such as specific resistivity for various types of GB. This section also contains the analytical equation derived from our numerical simulations. Last Section IV presents the conclusion and the summary of this work.

\section{SiMULATION APPROACH}

\section{A. Generation of atomistic structures of twin $G B S$}

All simulations in this work are performed with the commercial software Atomistix Tool Kit from Synopsys QuantumWise [10]. All simulations are based on the PerdewBurke-Ernzerhof version of the Generalized Gradient Approximation (GGA) exchange-correlation functional [11]. The criteria of the maximum force on an atom is $10^{-2} \mathrm{eV} / \AA$. The density mesh cutoff is taken to be 200 Hartree. As the first step, we geometrically optimise the atomistic structure of bulk $\mathrm{Cu}$ with the face centered cubic (FCC) structure. The calculated lattice parameter from our numerical simulations is $3.637 \AA$, which is in good agreement with the experimental value of 3.615 $\AA$ [12].

Another program called GBstudio [13] is used to find the transport direction of each grain and the angle between two sides for each GB. Based on this information, we construct the initial atomistic structures and we perform geometrical optimization by using the DFT method and the GGA functional. Fig. 1 shows the example atomistic structure of the $\Sigma 3$ GB (see case \#1 in Table 2). The explicit atomistic layers on either side of each grain boundary are fully optimized. The left-side and the right-side grains (see Fig. 1) have three atomic layers. The main reason for this is that exactly three layers were demonstrated to be the optimal number of layers needed to achieve reliable results [7].

\section{B. Transport simulations}

A DFT-NEGF method with the GGA functional is employed for the transport simulation in this work. Using the optimized atomistic structure of the GB, we build the device structure including left and right electrodes as shown in Fig. 1. It is assumed that the left and the right electrodes are semi-infinite which means that left-side and right-side of the grains are repeated periodically in the opposite direction from the GB interface. Adding electrodes to the atomistic structure is mandatory for using the NEGF framework in order to be able to evaluate the total resistance of this structure and more importantly the specific resistance for each GB.

Since the length of metal wires in the interconnects is much longer than a grain size, we assume that the electric field in this system is very small. Thus, we do not apply an external voltage on the left and the right electrodes during our transport simulations. Hence, the bias between two electrodes is equal to $0 \mathrm{~V}$ and the transmission spectrum is evaluated. The electronic temperature of the system is $300 \mathrm{~K}$ in all simulations.

Fig. 2 shows the calculated transmission spectrum of the $\Sigma 3$ GB shown in Fig. 1 as an example. From the data presented in

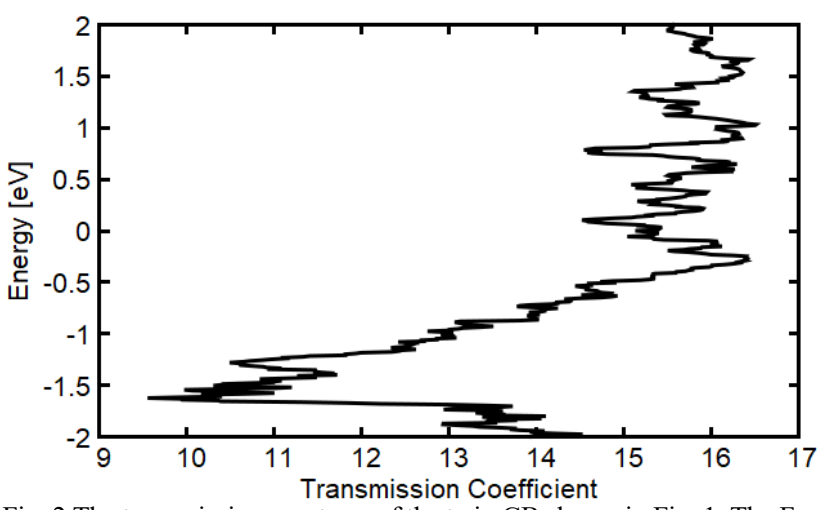

Fig. 2 The transmission spectrum of the twin GB shown in Fig. 1. The Fermilevel is set to $0.0 \mathrm{eV}$.

TABLE I. THE CALCULATED RESISTIVITY OF BULK CU WITH DIFFERENT TRANSPORT DIRECTIONS.

\begin{tabular}{|c|c|}
\hline Transport direction & Resistivity $(\mu \Omega \mathrm{cm})$ \\
\hline 100 & 2.843 \\
\hline 110 & 6.470 \\
\hline 111 & 2.298 \\
\hline 012 & 2.199 \\
\hline 031 & 2.218 \\
\hline $04 \overline{3}$ & 2.188 \\
\hline $2 \overline{1} 2$ & 2.261 \\
\hline $2 \overline{1} \overline{1}$ & 2.278 \\
\hline $2 \overline{2} \overline{1}$ & 2.261 \\
\hline $1 \overline{1} 4$ & 2.249 \\
\hline 023 & 2.203 \\
\hline $1 \overline{2} 1$ & 2.271 \\
\hline 041 & 2.219 \\
\hline $1 \overline{1} 3$ & 2.245 \\
\hline
\end{tabular}

Fig. 2, we are able to calculate the ballistic conductance ( $G_{b a l}$ ) of the system by using the equation below:

$$
G_{b a l}=\frac{1}{R_{b a l}}=G_{0} \int T(E) \frac{d E}{k_{B} T_{e}} \frac{e^{\frac{E-E_{F}}{k_{B} T_{e}}}}{\left(1+e^{\frac{E-E_{F}}{k_{B} T_{e}}}\right)^{2}}
$$

where $G_{0}, T, k_{B}, T_{e}$, and $E_{F}$ are the quantum conductance, the transmission coefficient, the Boltzmann constant, the electronic temperature, and the Fermi-level, respectively. The $G_{b a l}$ is an important parameter which is inversely proportional to the ballistic resistance.

\section{Calculation of the specific resitivity}

The resistance induced by GBs $\left(R_{G B}\right)$ is determined from the ballistic resistance of the atomistic structures with GBs $(R)$ and the idealized ballistic resistance of the structure $\left(R_{\text {ideal }}\right)$ as

$$
R_{G B}=R-R_{\text {ideal }} \text {. }
$$

Herein, $R_{\text {ideal }}$ indicates the resistance of the device structure with the GB assuming that all electrons can flow from the left 
TABLE II. SPECIFIC RESISTIVITY FOR DIFFERENT CSLS AND TRANSPORT DIRECTIONS.

\begin{tabular}{|c|c|c|c|c|c|}
\hline \multirow{2}{*}{$\begin{array}{c}\text { No. } \\
\text { (Group) }\end{array}$} & \multirow{2}{*}{$\begin{array}{l}\mathrm{CSL} \\
(\Sigma)\end{array}$} & \multicolumn{2}{|c|}{$\begin{array}{l}\text { Transport direction } \\
\text { of each grain }\end{array}$} & \multicolumn{2}{|c|}{$\begin{array}{c}\text { Specific resistivity } \\
{\left[\mathrm{p} \Omega \mathrm{cm}^{2}\right]}\end{array}$} \\
\hline & & $\begin{array}{l}\text { Left- } \\
\text { side }\end{array}$ & $\begin{array}{l}\text { Right- } \\
\text { side }\end{array}$ & $\begin{array}{l}\text { In this } \\
\text { work }\end{array}$ & Literatures \\
\hline $1(2)$ & \multirow{5}{*}{3} & {$[100]$} & {$[2 \overline{1} 2]$} & 1.090 & \\
\hline $2(1)$ & & [111] & [111] & 0.267 & $\begin{array}{c}0.170 *[16] \\
0.158[7] \\
0.156[14] \\
0.202[8]\end{array}$ \\
\hline $3(1)$ & & {$[2 \overline{1} \overline{1}]$} & {$[1 \overline{2} 1]$} & 2.606 & \\
\hline $4(3)$ & & {$[1 \overline{1} 0]$} & {$[0 \overline{1} 1]$} & 17.877 & \\
\hline $5(3)$ & & [110] & {$[411]$} & 5.506 & \\
\hline $6(2)$ & \multirow{4}{*}{5} & {$[100]$} & {$[100]$} & 3.066 & \\
\hline $7(1)$ & & [012] & [021] & 1.352 & $\begin{array}{c}1.885[8] \\
1.49[7] \\
1.759[14] \\
\end{array}$ \\
\hline $8(1)$ & & [031] & {$[03 \overline{1}]$} & 1.658 & \\
\hline $9(2)$ & & [010] & {$[04 \overline{3}]$} & 0.966 & \\
\hline $10(1)$ & 9 & {$[2 \overline{2} \overline{1}]$} & {$[2 \overline{2} 1]$} & 1.536 & $\begin{array}{c}1.75[7] \\
1.82[14] \\
\end{array}$ \\
\hline $11(1)$ & 11 & [1]̄̄3] & [1]13] & 0.591 & $\begin{array}{c}0.75[7] \\
0.64[14]\end{array}$ \\
\hline $12(1)$ & \multirow{2}{*}{$13 a$} & [023] & [032] & 2.461 & $\begin{array}{c}2.41[7] \\
2.01[14]\end{array}$ \\
\hline $13(2)$ & & {$[100]$} & {$[100]$} & 2.717 & \\
\hline $14(1)$ & \multirow{2}{*}{$17 \mathrm{a}$} & {$[041]$} & {$[04 \overline{1}]$} & 1.810 & 2.01 [7] \\
\hline $15(2)$ & & [100] & {$[100]$} & 3.339 & \\
\hline $16(2)$ & $25 a$ & {$[100]$} & {$[100]$} & 1.850 & \\
\hline
\end{tabular}

(right) electrode to the right (left) electrode without any scatterings. In [7] and [14], the resistance of bulk $\mathrm{Cu}\left(R_{\text {bulk }}\right)$ is used instead of $R_{\text {ideal }}$. However, the ballistic $R_{\text {bulk }}$ has a dependency on the transport direction as shown in Table I, where the calculated resistivity's of bulk $\mathrm{Cu}$ with different transport directions are summarized. For the calculation of the resistivity, we assume that the mean free path of bulk $\mathrm{Cu}$ is $40 \mathrm{~nm}$ regardless of the transport direction. This table shows that the resistivity varies from 2.188 to $6.470 \mu \Omega \mathrm{cm}$ which is very close to the previously reported experimental value of $1.68 \mu \Omega \mathrm{cm}$ [15]. Moreover, based on the data presented in Table I we can conclude that the ballistic resistances of the left-side and the right-side of the grains may be different which could have an impact on the correct estimation of the specific resistance.

In order to calculate the specific resistance more accurately, we calculate $R_{\text {ideal }}$ through several steps as shown below;

Step 1. We calculate the transmission spectrums of bulk $\mathrm{Cu}$ with the left-side grain's transport direction $T_{L}$ and with the right-side grain's transport direction $T_{R}$ by using the DFT-NEGF framework.

Step 2. A new transmission spectrum $T_{\text {ideal }}$ is defined in all ranges of energy;

$$
T_{\text {ideal }}(E)=\min \left(T_{L}(E), T_{R}(E)\right),
$$

where $\min (a, b)$ is a function used to find a smaller value between $a$ and $b$.

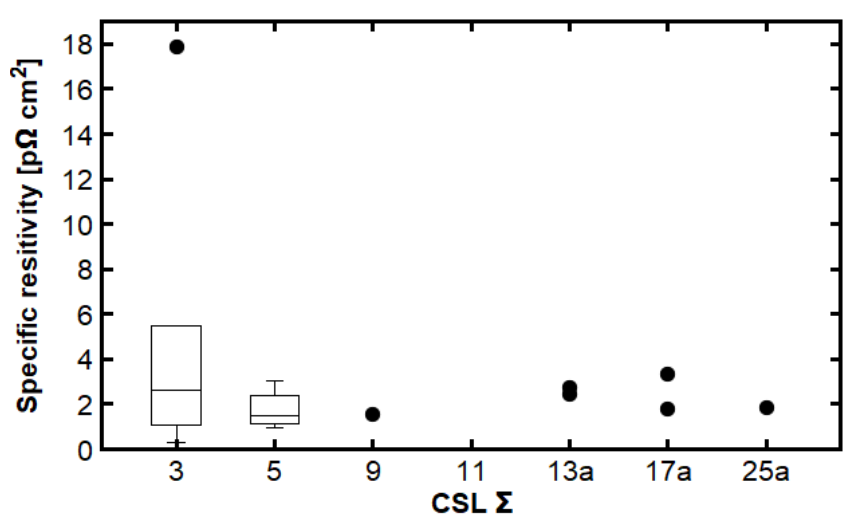

Fig. 3 The dependence of calculated specific resistivity of GBs on the coincident site lattice $(\mathrm{CSL}, \Sigma)$.

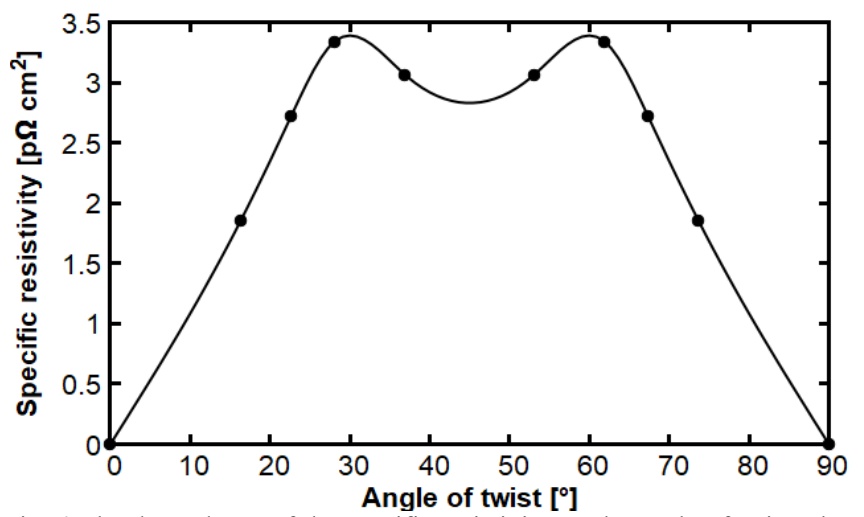

Fig. 4 The dependence of the specific resistivity on the angle of twist when the transport orientation of both grains is [100] (case 6, 12, 15, and 16 in Table II).

Step 3. $R_{\text {ideal }}$ can be calculated with $T_{\text {ideal }}$ by using Eq. 1 .

This shows that $R_{\text {ideal }}$ is very close to the larger transport grain resistance. Finally, the specific resistivity at the GB $\left(\gamma_{R}\right)$ can be obtained by using the equation below:

$$
\gamma_{\mathrm{R}}=R_{G B} \times A
$$

where $A$ is the cross-section area of the constructed atomistic structures of GBs.

\section{RESULTS AND DISCUSSION}

The calculated specific resistivity $\left(\gamma_{R}\right)$ for different CSLs and transport directions is summarized in Table II. The corresponding results obtained from previous studies are also displayed for comparison to our data. We find that our calculated results are in agreement with the previously reported research on this topic which indeed is very encouraging.

Fig. 3 shows the dependence of our calculated $\gamma_{R}$ value on the CSL. Unfortunately, we are not be able to find the correlation between $\gamma_{R}$ and CSL in the sample that we consider in this work. Hence, we believe that CSL cannot represent $\gamma_{R}$ and be used as a figure of merit. Moreover, in the case of $\Sigma 3 \mathrm{GBs}$, the variation of the $\gamma_{R}$ values is significant where the difference of minimum and maximum values of $\gamma_{\mathrm{R}}$ 's is $17.61 \mathrm{p} \Omega \mathrm{cm}^{2}$. 


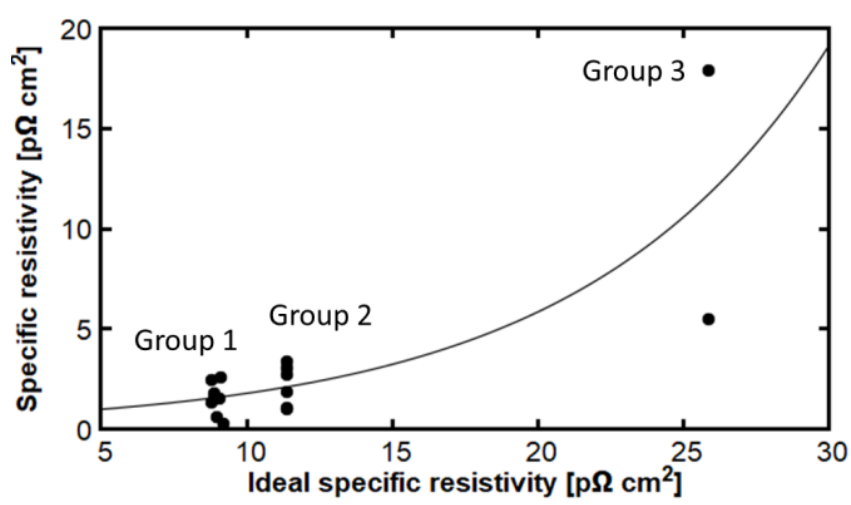

Fig. 5 The dependence of the specific resistivity on the specific resistivity of the ideal grain boundary $\left(R_{\text {ideal }} \times A\right)$.

In order to try to find correlation between the specific resistivity and the structure parameters, we investigate the specific resistivity as a function of the twist GBs when both grains have the [100] transport direction. Our results are shown in Fig. 4 and the following main points can be emphasised. Firstly, the simulation results follow the bimodal distribution with two equal peaks at near $30^{\circ}$ and $60^{\circ}$ angle and they show a mirror symmetrical shape around the $45^{\circ}$ twist angle, which is caused by the symmetry of the atomistic structure. Secondly, when the angle of twist is $26.87^{\circ}$, which corresponds to the $\Sigma 5$ GB (see case \#6 in Table II), the specific resistivity has the maximum value.

Fig. 5 describes the dependence of the specific resistivity of GBs on the ideal specific resistivity $\left(\gamma_{R}^{\text {ideal }}\right)$, which is calculated from $R_{\text {ideal }}$. It is important to point out that as the ideal specific resistivity increases, the specific resistivity of GBs also increases. Corresponding data is clustered in three groups - Group 1, Group 2 and Group 3 (see Fig. 5 and Table II). Hence, it can be concluded that the large ideal specific resistivity corresponds to the large resistivity of grains. Thus, all CSLs having the [110] transport direction, which shows the highest resistivity in Table I, correspond to Group 3 (see case \#4 and \#5 in Table II).

Based on the results presented above we derive an analytical equation based on the average values of each Group in Fig. 5:

$$
\gamma_{\mathrm{R}}=0.545 e^{0.1186 \gamma_{R}^{\text {ideal }}}
$$

By using equation (4), we believe that the specific resistivity $\left(\gamma_{R}\right)$ of other metals and materials can be predicted. For example, cobalt $(\mathrm{Co})$ which is one of the promising materials to replace $\mathrm{Cu}$ in future technologies as an interconnect [17], has an $\gamma_{R}^{\text {ideal }}$ for the hexagonal structure of $7.31 \mathrm{p} \Omega \mathrm{cm}^{2}$ [18]. Therefore, based on equation 4 we can predict that the specific resistivity $\gamma_{\mathrm{R}}$ for cobalt should be $1.297 \mathrm{p} \Omega \mathrm{cm}^{2}$. However, more research needs to be done in this area to validate further the transferability of our analytical equation. Indeed, this is one of the future research directions in our group.

\section{CONCLUSIONS}

In this work, we performed the Density Functional Theory simulations to calculate the specific resistivity of $\mathrm{GBs}$ in the $\mathrm{Cu}$ interconnects. We found that the coincident site lattice (CSL) model cannot represent the specific resistivity of the GB. Instead of the CSL, the transport direction is the most important parameter that strongly correlates with the specific resistivity. To summarise all our simulation results, we proposed the simple analytical model to predict the specific resistivity of the GB from the specific resistivity of bulk atomistic configuration. We believe that the proposed analytical equation and methodology could be extended and applicable to other systems and materials such as cobalt, for example. We are currently conducting research in this direction to validate our model for the cobalt interconnect.

\section{REFERENCES}

[1] R. H. Havemann and J. A. Hutchby, "High-performance interconnects: an integration overview”, Proc. the IEEE, vol. 89, no. 5, pp. 586-601, May 2001 .

[2] S. M. Rossnagel, and T. S. Kuan, "Alteration of Cu conductivity in the size effect regime”, J. Vac. Sci. Technol. B, vol. 22, pp. 240-247, Jan. 2004.

[3] W. Wu, S. H. Brongersma, M. Van Hove, and K. Maex, "Influence of surface and grain-boundary scattering on the resistivity of copper in reduced dimensions", Appl. Phys. Lett., vol. 84, no. 15, pp. 2838-2840, Apr. 2004.

[4] W. Steinhgl, G. Schindler, G. Steinlesberger, M. Traving, and M. Engelhardt, "Comprehensive study of the resistivity of copper wires with lateral dimensions of $100 \mathrm{~nm}$ and smaller”, J. Appl. Phys., vol. 97, 023706, 2005

[5] J. R. Lloyd, and J. J. Clement, "Electromigration in copper conductors", Thin Solid Films, vol. 262, no. 1-2, pp. 135-141, Jun. 1995.

[6] J. Lee et al. "Understanding Electromigration in Cu-CNT Composite Interconnects: A Multiscale Electro-Thermal Simulation Study", unpublished.

[7] M. César, D. Liu, D. Gall, and H. Guo, "Calculated Resistances of Single Grain Boundaries in Copper”, Phys. Rev. Applied, vol. 2, pp. 044007, Oct. 2014.

[8] T. Kim et al., "Large Discrete Resistance Jump at Grain Boundary in Copper Nanowire", Nano Lett., vol. 10, pp. 3096-3100, July 2010.

[9] M. Brandbyge, J. L. Mozos, P. Ordejón, J. Taylor, and K. Stokbro, "Density-functional method for nonequilibrium electron transport", Phys. Rev. B, vol. 65, pp. 165401, Mar. 2002.

[10] Atomistix Tool Kit version 2017.2, Synopsys QuantumWise A/S. Availabe at http://www.quantumwise.com. [Accessed June 20. 2018].

[11] J. P. Perdew, K. Burke, and M. Ernzerhof, "Generalized Gradient Approximation Made Simple", Phys. Rev. Lett., vol. 77, pp. 3865, Oct. 1996.

[12] H.W. King, CRC Handbook of Chemistry and Physics, vol. 83, p. 19. 2002,

[13] GBStudio ver $3.0 \quad$ Available at https://staff.aist.go.jp/h.ogawa/GBstudio/indexE.html. [Accessed June 20, 2018]

[14] D. Valencia, E. Wilson, Z. Jiang, G. A. Valencia-Zapata, K. Wang, G. Klimeck, and M. Povolotskyi, "Grain-Boundary Resistance in Copper Interconnects: From an Atomistic Model to a Neural Network", Phys. Rev. Appl., vol. 9, pp. 044005, Apr. 2018.

[15] R. A. Matula, "Electrical resistivity of copper, gold, palladium, and silver", J. Phys. Chem. Ref. Data., vol. 8, no. 4, pp. 1147-1298, 1979.

[16] L. Lu, Y. Shen, X. Chen, L. Qian, and K. Lu, "Ultrahigh strength and high electrical conductivity in copper", Science, vol. 304, pp. 422-426, Mar. 2004.

[17] N. Bekiaris et al.," Cobalt Fill for Advanced Interconnects", Proc. 2017 IEEE International Interconnect Technology Conference (IITC).

[18] D. Gall, "Electron mean free path in elemental metals", J. Appl. Phys., vol. 119, pp. 085101, Feb. 2016. 